\title{
Taking advantage of coronavirus disease 2019 (COVID-19) to improve hospital models of care: Are we moving in the right direction?
}

\author{
Raffaella Gualandi RN, PhD and Anna De Benedictis RN, PhD (1) \\ Department of Nursing, Campus Bio-Medico University of Rome, Rome, Italy
}

To the Editor-The current pandemic is disrupting most industries and sectors, with an epicenter in the healthcare sector. With no substantial experience in managing an epidemic, hospitals were involved by first responding to a sudden influx of patients with severe and highly contagious respiratory conditions. They reconverted spaces and environments for coronavirus disease 2019 (COVID-19) patient care. Finally, they contributed to vaccination implementation. Hospital managers have had to reorganize hospital access, spaces, and patient flow, as well as review all hospital policies, to facilitate the treatment of all patients with different pathologies in so-called "COVIDfree" spaces. ${ }^{1}$ The increase in the volume and complexity of patient care, the sudden and unpredictable decrease in staff, and the lack of patient support from family members and caregivers are just some of the main variables that hospital managers have had to handle. ${ }^{2}$

During this COVID-19 crisis, all of the professionals involved in health care have acted in view of the greatest good for the greatest number of people. Making patients and workers safe is a main driver of hospital management. Consequently, the hospital model of care has also progressively changed to balance the different priorities of the safety of patients and healthcare professionals with the medical treatments provided. A model of care can be defined as "the way health services are delivered. It outlines best practice care and services for a person, population group, or patient cohort as they progress through the stages of a condition, injury, or event. It aims to ensure people get the right care, at the right time, by the right team and in the right place." 3 During this pandemic, necessary safety rules introduced in inpatient care have generated unmet and perhaps still hidden needs among patients. For example, how can the patient correctly understand the therapeutic plan if she or he does not have her or his informal caregiver at hand? How can a patient overcome worry while waiting alone for surgery or chemotherapy? How can family members have constant news about their loved one's postoperative progress? How can the patient receive what she or he needs from home for prolonged hospitalization?

Substantial changes have gradually occurred in the hospital model of care resulting from medical science and technological development (Fig. 1). Past models probably would not have responded to the rapidly changing needs of modern health systems. Hospitals were organized into static, self-sufficient departments where all resources were dedicated to treating patients from a disease-centered perspective. But this low-

Author for correspondence: Anna De Benedictis, E-mail: a.debenedictis@unicampus.it Cite this article: Gualandi $\mathrm{R}$ and De Benedictis A. (2022). Taking advantage of coronavirus disease 2019 (COVID-19) to improve hospital models of care: Are we moving in the right direction?. Infection Control \& Hospital Epidemiology, 43: 1283-1284, https://doi.org/10.1017/ice.2021.210 reactivity model with poor central coordination would have resulted in failure to deliver essential care to patients.

Improving clinical outcomes and making hospital resources more efficient, medical super-specialization and the exponential advance of technology have generated specialized care and multifunctional and shared high-tech services. Hospitals are organized into specialist units (eg, cardiology, orthopedics, nephrology), with dynamic resource allocation according to the demand. Because of the centralized coordination of the main hospital assets (beds, operating theaters, and clinics), response to the COVID-19 crisis has been relatively rapid, converting the various hospital services according to priority, reallocating resources where they were most needed, and allowing many hospital units to survive.

However, the multiple pathologies of patients with an increasingly advanced average age affecting the personal and social sphere at all levels of ordinary life was already putting pressure on the current model. The nature of modern health care, focused only on clinical knowledge with no time for administrative or systems thinking, can make fluid collaboration between professionals a challenge. A consequent fragmentation of the hospital journey of patients who have to pass from one service to another, which all too often do not communicate with each other, is just one of the weaknesses of this model. Even though hospital managers are progressively considering organizing hospitals into homogenous areas according to patient care needs, the experiment of dividing a hospital according to intensity of care seems to have not yet taken hold. ${ }^{4}$ By centralizing the control and allocation of hospital resources, minimizing hospital access to the bare essentials, and prioritizing the treatments to be provided, the virus appears to have halted its spread within the hospital. However, we still do not know how the hospitalized patient is experiencing this new scenario.

Although "patient-centered" medicine focuses on the patient's health needs and outcomes, what hospital model of care should we now pursue ${ }^{5}$ The new model should integrate the concept of "disease," meaning a group of signs and symptoms, with that of "illness," which includes personal expectations and social context, and with that of "needs," which includes the patient's experience of her or his care pathway. Hospital managers have to move from a narrow focus on individual patients' disease states to a broad focus on health-related social factors (ie, social and family relationships, proper nutrition, accessing the correct information for health and social well-being) by creatively engaging patients. ${ }^{6}$ Moreover, this pandemic has highlighted the important role that patients have in managing their own health, including understanding and acting on health information.

In conclusion, the extreme situation caused by the pandemic is a rare learning opportunity to improve our hospital model of care. The pandemic crisis response was a clear example of how 


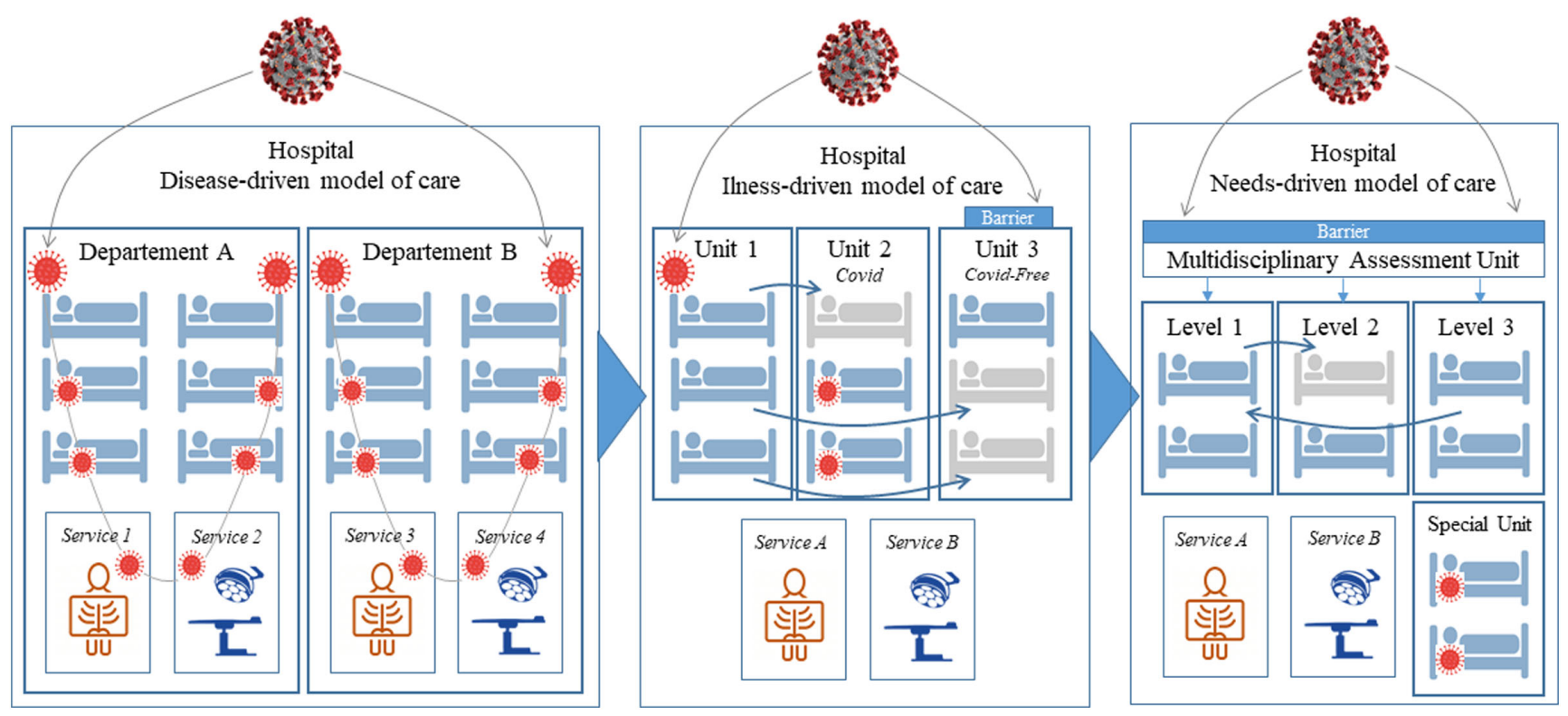

Fig. 1. Main transitions of the hospital model of care and response capacity to the spread of COVID-19.

health is achieved with cross cutting and coordinated interventions that go beyond medical treatment. The COVID-19 compelled us to run, and now we need to focus the coordinates in the right direction.

Author contributions. RG and ADB contributed to the literature review and to the final version of the manuscript. The first draft of the manuscript was written by RG.

Financial support. No financial support was provided relevant to this article.

Conflicts of interest. All authors report no conflicts of interest relevant to this article.

\section{References}

1. Bearman G, Pryor R, Albert H, et al. Novel coronavirus and hospital infection prevention: Preparing for the impromptu speech. Infect Control Hosp Epidemiol 2020;41:592-593.
2. Rosenbaum L. Facing COVID-19 in Italy-ethics, logistics, and therapeutics on the epidemic's front line. N Engl J Med 2020;382:1873-1875.

3. Understanding the process to develop a model of care. A practical guide on how to develop a model of care at the agency for clinical innovation, version 1.0. Agency For Clinical Innovation website. https://www.aci.health.nsw.gov.au/ __data/assets/pdf_file/0009/181935/HS13-034_Framework-DevelopMoC_D7. pdf. Published May 2013. Accessed February 19, 2020.

4. Liguori I, Russo G, Aran L, et al. Acute-care hospital at different levels of intensity: the role of geriatrician. Aging Clin Exp Res 2018;30:703-712.

5. Bardes CL. Defining "patient-centered medicine." $N$ Engl J Med 2012;366:782-783.

6. Bate P, Robert G. Experience-based design: from redesigning the system around the patient to codesigning services with the patient. Qual Saf Health Care 2006;15:307-310.

\title{
Saliva and its potential in coronavirus disease 2019 (COVID-19) cannot be ignored: A point of view
}

\author{
Sukhvinder Singh Oberoi BDS, MDS$^{1}$ (1), Shabina Sachdeva BDS, MDS², Shibani Grover BDS, MDS $^{3}$ and \\ Vivek Sharma BDS, MDS ${ }^{3}$
}

${ }^{1}$ Public Health Dentistry, ESI Dental College and Hospital, Sector 15, Guru Gobind Singh Indraprastha University, Rohini, Delhi, India, ${ }^{2}$ Prosthodontics, Faculty of Dentistry, Jamia Millia Islamia, Jamia Nagar, Delhi, India and ${ }^{3}$ Conservative Dentistry and Endodontics, ESIC Dental College, Guru Gobind Singh Indraprastha University, Rohini, Delhi, India

Author for correspondence: Sukhvinder Singh Oberoi, E-mail: drsukhvinder@gmail. com

Cite this article: Oberoi SS, et al. (2022). Saliva and its potential in coronavirus disease 2019 (COVID-19) cannot be ignored: A point of view. Infection Control \& Hospital Epidemiology, 43: 1284-1285, https://doi.org/10.1017/ice.2021.211
To the Editor-Recent research has shown that $\sim 1.2 \times 10^{8}$ infective copies/mL of severe acute respiratory coronavirus virus 2 (SARS$\mathrm{CoV}-2)$ virus can be found in the salivary samples of coronavirus disease 2019 (COVID-19)-positive patients. ${ }^{1}$ The saliva and 